[Technical Paper]

\title{
Study on Gate Bias Voltage Application Conditions to Prevent the Knee Voltage Shift of the SiC-MOSFET Body Diode Depending on Gate Bias Voltage during Transient Temperature Measurements
}

\author{
Fumiki Kato*, Shinji Sato*, Hidekazu Tanisawa**, Hiroshi Hozoji*, Hiroshi Yamaguchi*, and Hiroshi Sato* \\ *National Institute of Advanced Industrial Science and Technology (AIST), Umezono 1-1-1, Tsukuba, Ibaraki 305-8560, Japan \\ **Sanken Electric Co., Ltd., 3-6-3 Kitano, Niiza-shi, Saitama 352-8666, Japan
}

(Received August 23, 2019; accepted March 27, 2020, published May 22, 2020)

\begin{abstract}
This paper reports a method for determining the gate bias voltage condition during transient temperature measurement using a SiC-MOSFET body diode. The Vg-Vsd characteristic of the device was investigated. There exists a plateau where Vsd does not change with changes in Vg. In this plateau, transient temperature measurement using a body diode is possible under the gate bias condition. This method was applied to three devices with different characteristics. The results confirmed that transient temperature measurement is possible by selecting appropriate Vg values according to the relevant device characteristics. The transient temperature of each module in which three devices (Device A, Device B1, and Device B2) are mounted in the same structure package are measured. The three transient temperature graphs agree well, and the three structure function graphs are also in good agreement. Thus, the proposed method allows the rapid determination of parameters of a transient thermal analysis for an unknown device.
\end{abstract}

Keywords: Transient Temperature Measurement, Transient Thermal Analysis, SiC-MOSFET, Body Diode, Knee Voltage Shift, Gate Bias Voltage

\section{Introduction}

$\mathrm{SiC}$ power devices have gained considerable attention because they can be operated at higher current density and operating temperature than those of the conventional Si power devices. [1-4] When a power device can be operated at a high temperature, it is possible to minimize the cooling equipment required for the power converter. Accurate thermal design technology and thermal property evaluation technology are necessary to improve the power density and realize stable and reliable operation of the power module. Thus, the transient thermal analysis method is considered a promising technology for evaluating the thermal resistance of a power module in an actual package. $[5,6]$ The accuracy of this analytical method depends on how accurately the cooling transient temperature is measured. The junction temperature of a $\mathrm{SiC}-$ MOSFET is measured by considering the temperature dependency of the I-V characteristic of the intrinsic body diode existing between the drain and source.[7] Generally, the gate and drain are connected directly to ensure that the gate potential does not become unstable during temperature measurement using the body diode. However, temperature measurement is affected by influence of the knee voltage shift when a SiC-MOSFET body diode is used as the temperature sensor.[8] Therefore, we have proposed a method for avoiding the knee voltage shift by negative gate-source bias during transient temperature measurement.[9] However, we found that the required bias voltage differs depending on the type of the SiCMOSFET product. We propose a method to set the appropriate gate bias.

\section{Knee Voltage Shift Depending on the Gate Bias} Voltage in the Body Diode of a SiC-MOSFET

To measure the junction temperature of the MOSFET, the temperature dependency of the I-V characteristic of the body diode is used. Figure 1 shows a schematic circuit diagram for transient temperature measurement. The junction temperature is measured by applying a constant current through the body diode (from the source to the drain) 
and measuring the built-in voltage between the source and drain. In the case of a Si-MOSFET, usually, the gate and drain are directly connected. However, a power supply must be connected between the gate and source to ensure that the gate potential does not become unstable when using the body diode of the SiC-MOSFET. Figure 2 shows the I-V characteristics of a SiC-MOSFET body diode for gate voltages of $0 \mathrm{~V},-2.0 \mathrm{~V},-4.0 \mathrm{~V},-6.0 \mathrm{~V}$, and $-10.0 \mathrm{~V}$. This figure clearly shows that a knee voltage shift occurs depending on the gate bias. If the gate and drain are directly connected, the gate bias will change according to the change in the forward voltage of the body diode, which is caused by changes in the temperature. For example, when the temperature-sensing current is set to $0.10 \mathrm{~A}$, the gate bias is about $-2.6 \mathrm{~V}$, which is the same as the forward voltage (Vsd) of the body diode. However, since this Vsd fluctuates with temperature during transient thermal testing, the gate bias also fluctuates accordingly. The I-V characteristics do not vary with a gate bias less than $-4.0 \mathrm{~V}$, though they are affected when the gate bias is set between 0 and $-4.0 \mathrm{~V}$. When using the body diode of a SiCMOSFET with such a characteristic, if the gate bias is exceeding $-4.0 \mathrm{~V}$, there is a possibility of errors in the transient temperature measurement because of the influ-

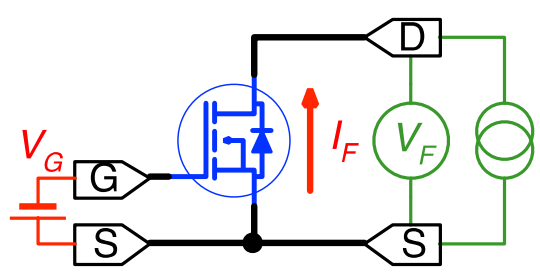

Fig. 1 Transient temperature measurement setup using the body diode of a SiC-MOSFET.

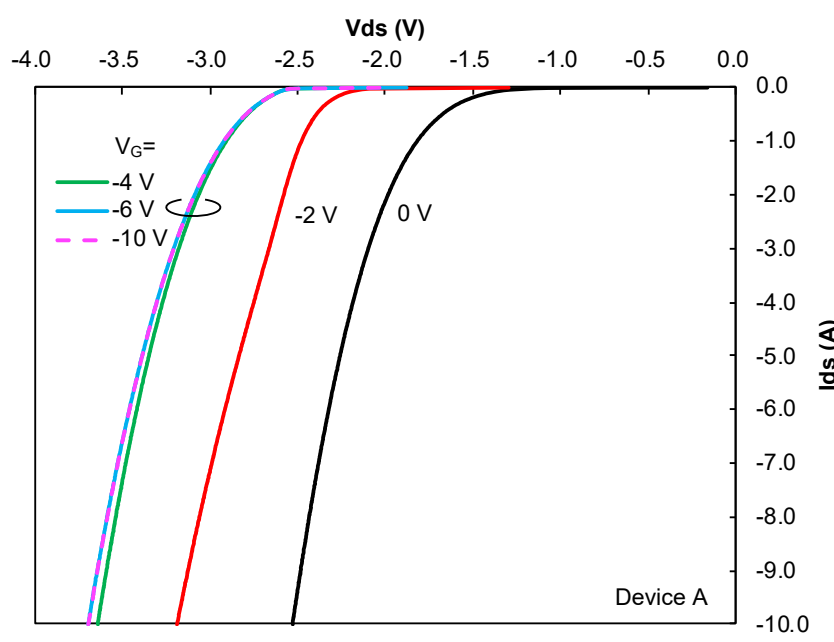

Fig. 2 Forward I-V characteristics of the body diode of $\mathrm{SiC}-$ MOSFET (for Device A) according to the gate bias condition. ence of the variation in the $\mathrm{I}-\mathrm{V}$ characteristic. Thus, the condition of the conventional Si-MOSFET (gate-drain short) cannot be applied for transient temperature measurement when using the SiC-MOSFET.

\section{Preventing Knee Voltage Shift Depending on the Gate Bias Voltage during Transient Temperature Measurement for the SiC-MOSFET}

The voltage at which the knee voltage shift occurs is investigated further in this study. In order to measure the temperature of the device, the device was used beyond normal rating conditions. Therefore, the names of the manufacturers of the devices are not disclosed herein, and devices with different manufacturers are simply labeled Device A and Device B. Since the characteristics of Device $\mathrm{B}$ varied depending on the manufacturing lot, it is introduced as Device B1 and Device B2.

First, the voltage range that could be applied to the gate was investigated. The leakage current when applying a voltage from $0 \mathrm{~V}$ to $-25 \mathrm{~V}$ between the gate and source of the devices were measured by using a semiconductor parameter analyzer. Figure 3 shows the measurement results of the gate current (leakage current) with respect to the gate voltage for Device A, Device B1, and Device B2. A leakage current was observed below $-20 \mathrm{~V}$ or less for both devices. The rated gate voltage range of the SiCMOSFET is $-10 \mathrm{~V}$ to $25 \mathrm{~V}$ for these products. Therefore, to avoid the destruction of the gate structure, it was decided that the gate voltage should not be set below $-20 \mathrm{~V}$.

Then, the voltage between source and drain (Vsd) relative to the voltage between gate and source $(\mathrm{Vg})$ was investigated for a drain current of $0.10 \mathrm{~A}$. This condition is

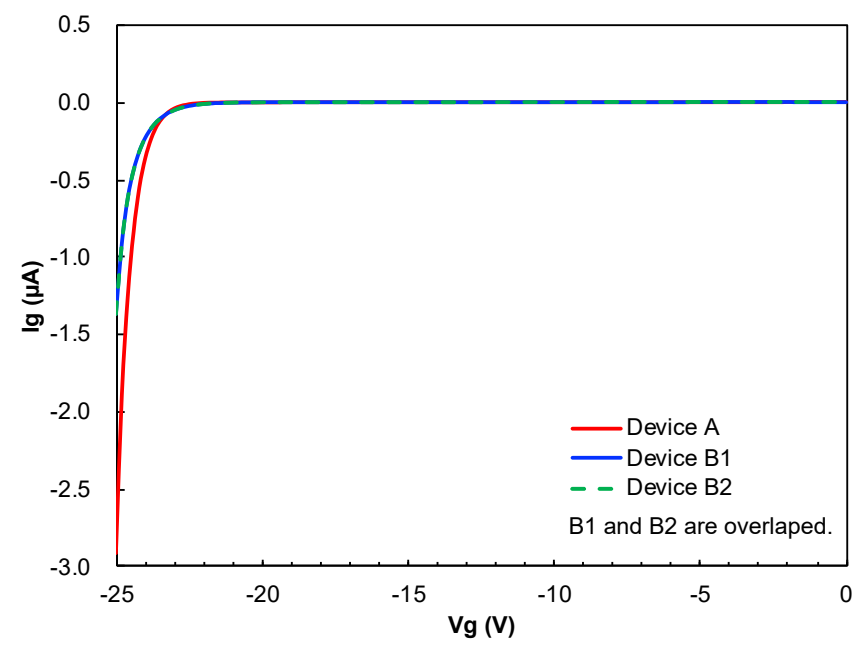

Fig. 3 Gate leak current according to gate bias for Device A and Device B. 
identical to the condition of applying a current for transient thermal testing. Figure 2 implies that in order to properly measure the temperature of the device using the body diode, it is necessary to set Vg such that Vsd does not change with changes in $\mathrm{Vg}$. The measurement results of Device A, Device B1, and Device B2 are shown in Fig. 4. Figure 5(a) shows the magnified view of a part of Fig. 4, and Fig. 5(b) shows the first derivative of Vg-Vsd. From these figures, it is possible to determine the change in Vsd with respect to $\mathrm{Vg}$. In case of Device $\mathrm{A}$, there was no change in the drain voltage for a gate bias of $-3.5 \mathrm{~V}$ or less. In case of Device B1, the drain voltage is constant for gate bias ranging from $-7.5 \mathrm{~V}$ to $-9.5 \mathrm{~V}$. However, when the gate bias ranges from $-9.5 \mathrm{~V}$ to $-17 \mathrm{~V}$, the drain voltage decreases according to gate bias. Then, the drain voltage becomes constant again in for gate bias ranging from -17

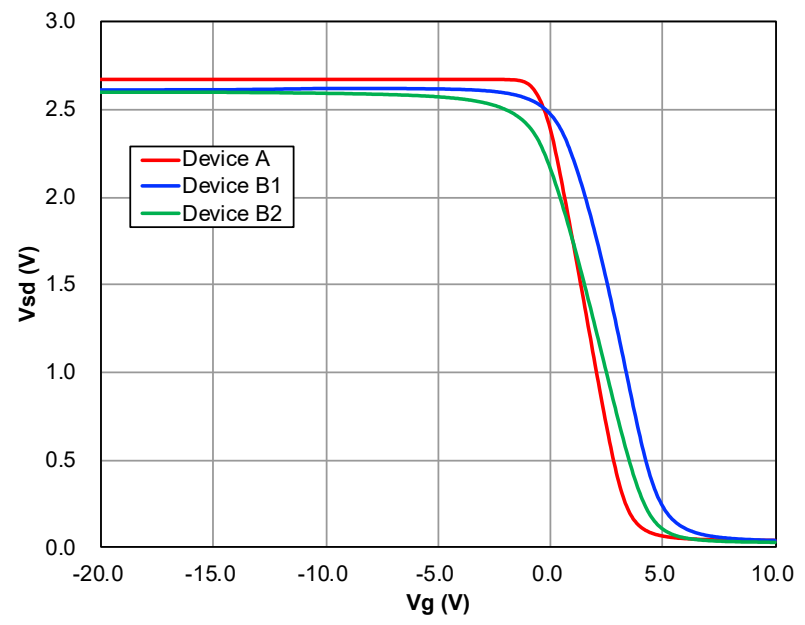

Fig. $4 \mathrm{Vg}-\mathrm{Vsd}$ characteristics with a source-drain current of 0.10 A for Device A, Device B1, and Device B2.
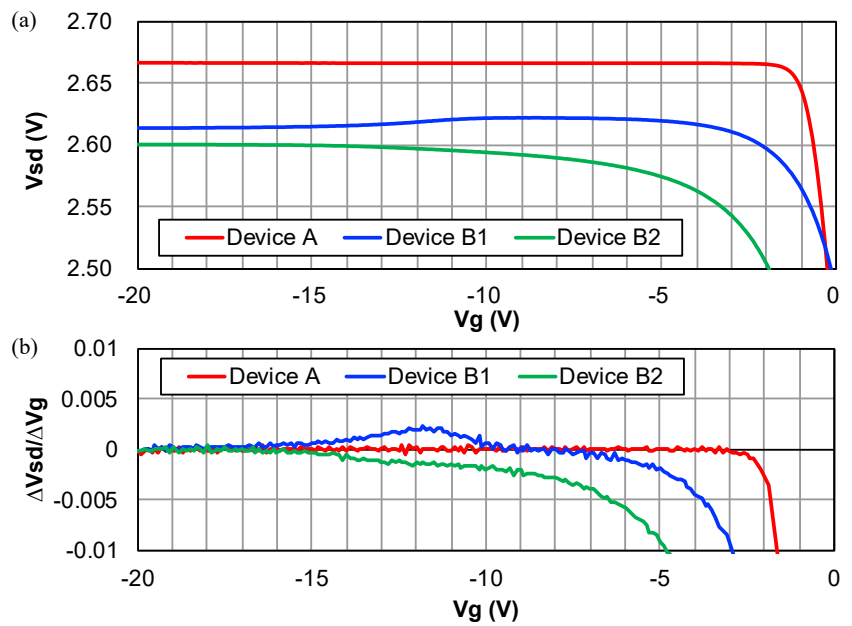

Fig. 5 (a) is a magnified view of a part of the Vg-Vsd characteristics and (b) is the first derivative of $\mathrm{Vg}-\mathrm{Vsd}$ characteristics for Device A, Device B1, and Device B2.
V to $-20 \mathrm{~V}$. For Device B2, the drain voltage was constant only when for gate bias between $-16 \mathrm{~V}$ and $-20 \mathrm{~V}$.

The Vg-Vsd characteristics of Device A and Device B1 and Device B2 are significantly different. This is expected because the manufacturing parameters of each manufacturer should be different. Further, differences were also observed between Devices B1 and B2, though they were manufactured by the same manufacturer. As seen in Fig. 4, the point at which Vg becomes positive and Vsd drops significantly, that is, the threshold voltage (Vth) of the MOSFET, differs greatly among devices. Although the cause has not been identified, it is possible that the devicemanufacturing parameters may have changed. These results indicate that the transient temperature can be correctly measured by setting the gate bias to $-3.5 \mathrm{~V}$ or less, $-15 \mathrm{~V}$ or less, $-16 \mathrm{~V}$ or less for Device A, Device B1, and Device B2, respectively.

\section{Transient Temperature Measurement Using the Body Diode of a SiC-MOSFET}

In order to confirm that temperature measurement can be performed correctly by setting a specific gate bias, transient temperature measurements were carried out. The modules were assembled using Device A, Device B1, or Device B2 and were mounted identical packages exhibiting the same structures and material specifications. We can conclude that the transient temperature measurement was properly performed with respect to each device if the results for the three aforementioned devices were identical. In each module, the $\mathrm{SiC}-\mathrm{MOSFET}$ is bonded to the silicon nitride active metal brazed copper ( $\mathrm{SiN}-\mathrm{AMC}$ ) substrates with a solder, and the substrate and base plate are joined by the solder, as shown in Fig. 6. The baseplate is manufactured using copper-tungsten. The SiC-MOSFET

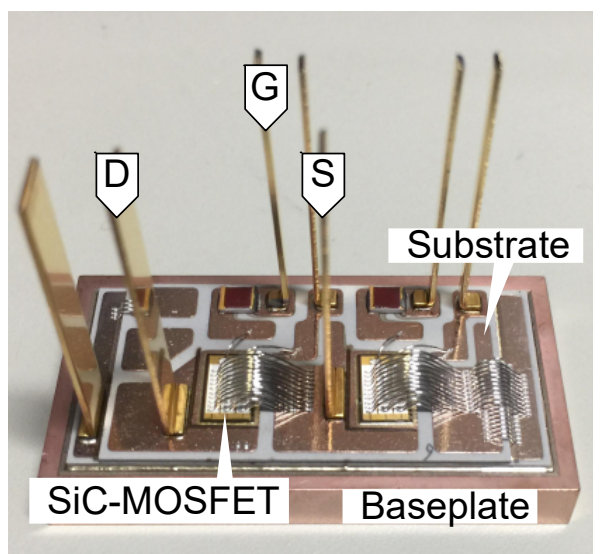

Fig. 6 Packaged module assembled with a SiC-MOSFET for transient temperature measurement. 
die size is $6.4 \times 4.0 \times 0.18 \mathrm{~mm}$ for Device $\mathrm{A}$ and $6.4 \times 4.0 \times$ $0.36 \mathrm{~mm}$ for Device B. The heating-up current was adjusted to $3.00 \mathrm{~A}$ (generating heat of approximately $10 \mathrm{~W}$ for each device) to ensure that the increase in junction temperature would become approximately $10^{\circ} \mathrm{C}$. The temperature-sensing current was set to $0.10 \mathrm{~A}$ to ensure that the heat generation effects can be ignored. A gate bias voltage of 0 to $-18 \mathrm{~V}$ was applied. The temperature sensitive parameters ( $\mathrm{K}$-factors) for converting the measured voltage into temperature are $-2.58 \mathrm{mV} / \mathrm{K},-2.61 \mathrm{mV} / \mathrm{K}$, and $-2.43 \mathrm{mV} / \mathrm{K}$ for Device A, Device B1, and Device B2, respectively.

The measurement results for Device A are presented in Fig. 7. In this graph, the horizontal axis denotes the elapsed time after the heating-up current was turned off, whereas the vertical axis denotes the temperature obtained from the measured voltage based on the K-factor. The subsequent data are the temperature obtained by considering $20 \mu$ s as the cut-off time ( $\left.\mathrm{t}_{\text {cut }}\right)$ due to the effects of the electrical noise generated by turning off the heating-up current. The four transient temperature response lines for gate biases of $-4.9 \mathrm{~V},-6.6 \mathrm{~V},-9.8 \mathrm{~V}$, and $-13.1 \mathrm{~V}$ overlap, but those for gate biases of $0 \mathrm{~V}$ and $-3.3 \mathrm{~V}$ did not overlap with other lines. For gate biases of $0 \mathrm{~V}$ and $-3.3 \mathrm{~V}$, the junction temperature increased at the first (despite the cooling transient) and then decreased. For a bias voltage of $-4.9 \mathrm{~V}$ or less, the graph becomes a monotonous descent curve, and it is understood that the cooling transient temperature can be measured appropriately. This is attributed to the fact that the $\mathrm{Vg}-\mathrm{Vsd}$ characteristic becomes a plateau for $-3.5 \mathrm{~V}$ or less, as seen in Fig. 5.

Figure 8 shows the transient temperature measurement results for Device B1. The inset in Fig. 8 presents the mag-

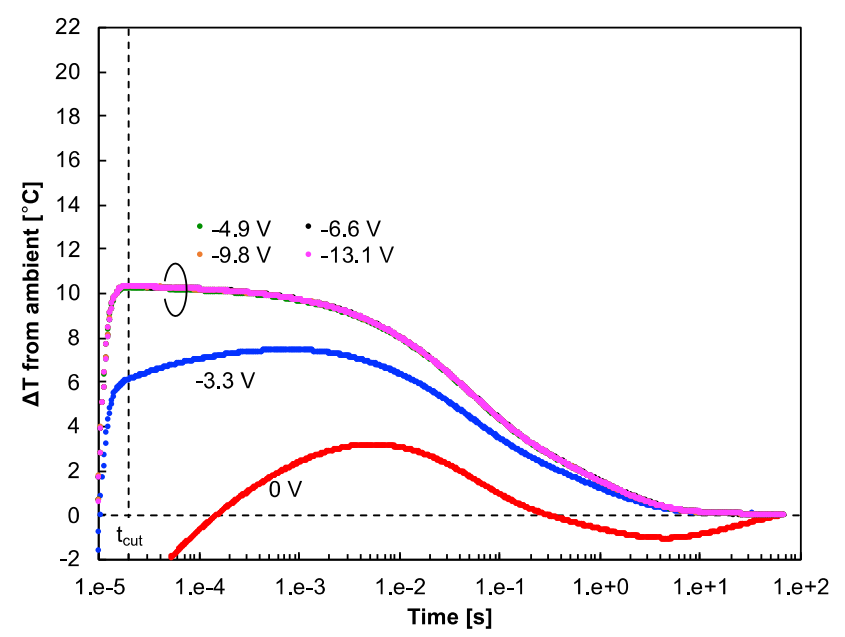

Fig. 7 Transient temperature of Device A for various gate biases. nified view of a portion of the graph. When the gate bias was $0 \mathrm{~V}$, the junction temperature increased initially and then decreased. The other graphs are monotonic decrease curves. However, graphs corresponding to a gate bias of $-3.6 \mathrm{~V}$ show a high initial temperature and are significantly different from the other graphs. Four transient temperature response lines corresponding to gate biases of $-7.2 \mathrm{~V}$, $-10.8 \mathrm{~V},-14.4 \mathrm{~V}$, and $-18.0 \mathrm{~V}$ are almost overlapping. The graphs from top to bottom correspond to gate biases of $-10.8 \mathrm{~V},-14.4 \mathrm{~V},-7.2 \mathrm{~V}$, and $-18.0 \mathrm{~V}$, and the difference between the maximum and minimum values of the four graphs is about $0.3^{\circ} \mathrm{C}$ at $20 \mu \mathrm{s}$. However, only the graph denoting a gate bias of $-7.2 \mathrm{~V}$ exhibits a different temperature trend when compared with those exhibited by the remaining graphs. Therefore, a bias of $-7.2 \mathrm{~V}$ was insufficient to suppress the voltage fluctuation. From the Vg$\Delta \mathrm{Vsd} / \Delta \mathrm{Vg}$ graph, the change amount is small in the region near $\mathrm{Vg}=-7.2 \mathrm{~V}$. However, the negative change continues; thus, the transient temperature measurement is considered to be influenced by the change in Vsd. In Device B1, the transient temperature could be measured properly by selecting the gate bias corresponding to the positive region of $\Delta \mathrm{Vsd} / \Delta \mathrm{Vg}$, more preferably the plateau of the $\mathrm{Vg}-\mathrm{Vsd}$ characteristic.

Figure 9 shows the measurement results of the transient temperature for Device B2. For gate biases of $0 \mathrm{~V},-3.6 \mathrm{~V}$, $-7.2 \mathrm{~V}$, and $-10.8 \mathrm{~V}$, the junction temperature temporarily rises and then decreases; this is not an appropriate cooling transient response.

Thus, it is possible to determine an appropriate gate bias necessary for transient temperature measurement by evaluating results of the $\mathrm{Vg}-\mathrm{Vsd}$ characteristics.

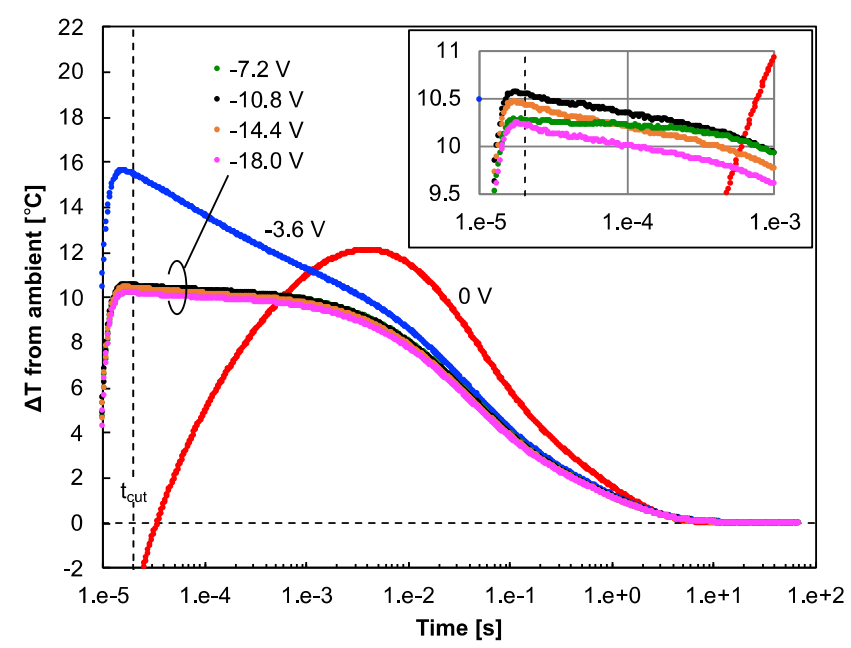

Fig. 8 Transient temperature of Device B1 for various gate biases Inset: The magnified view of a part Device B1 at the transient temperature. 


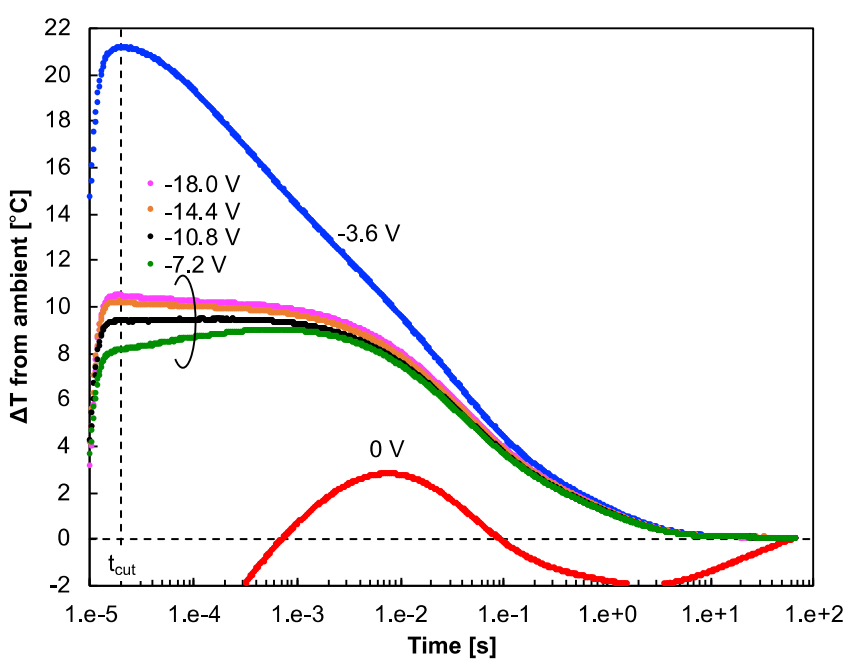

Fig. 9 Transient temperature of Device B2 for various gate biases.

\section{Structure Function of the Power Module}

In order to verify whether the transient temperature measurement results of each device are influenced by other device-dependent characteristics, a transient thermal analysis was performed. For example, a bias-stressinduced SiC MOSFET threshold voltage instability can affect the transient temperature measurements.[10] Thermal resistance analysis by induced transient method (TRAIT), which is called simply as transient thermal analysis, is used to calculate the time-constant spectrum (frequency domain) from the transient temperature (time domain) and derive a graph of the structure function with respect to the thermal resistance and heat capacity. $[5,6$, 11] If signals with different time constants are superimposed, even small differences that are difficult to distinguish in the time domain can be detected based on the structure function, which is the analysis result in the frequency domain.

The structure functions obtained based on the transient thermal analysis are shown in Figs. 10, 11, and 12, which correspond to those obtained based on the transient temperature in Figs. 7, 8, and 9, respectively. In Fig. 10, when the gate bias values are $-4.9,-6.6,-9.8$, and $-13.1 \mathrm{~V}$, the structure function graphs of Device A overlapped, as expected from the result of Fig. 7. In device B1, the three transient temperature response lines corresponding to the gate bias values of $-10.8,-14.4$, and $-18.0 \mathrm{~V}$ almost overlapped (Fig. 8). Correspondingly, the structure function graphs for the gate bias values of $-10.8,-14.4$, and $-18.0 \mathrm{~V}$ overlapped, as shown in Fig. 11. The graph for a gate bias of $-7.2 \mathrm{~V}$, where the transient temperature trend was different, did not overlap with the remaining graphs as

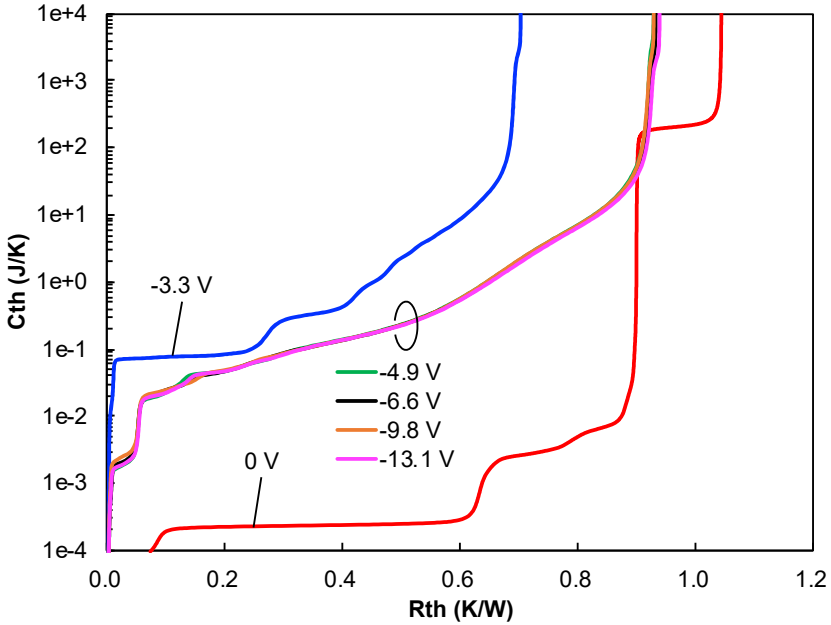

Fig. 10 Structure functions of Device A for various gate biases.

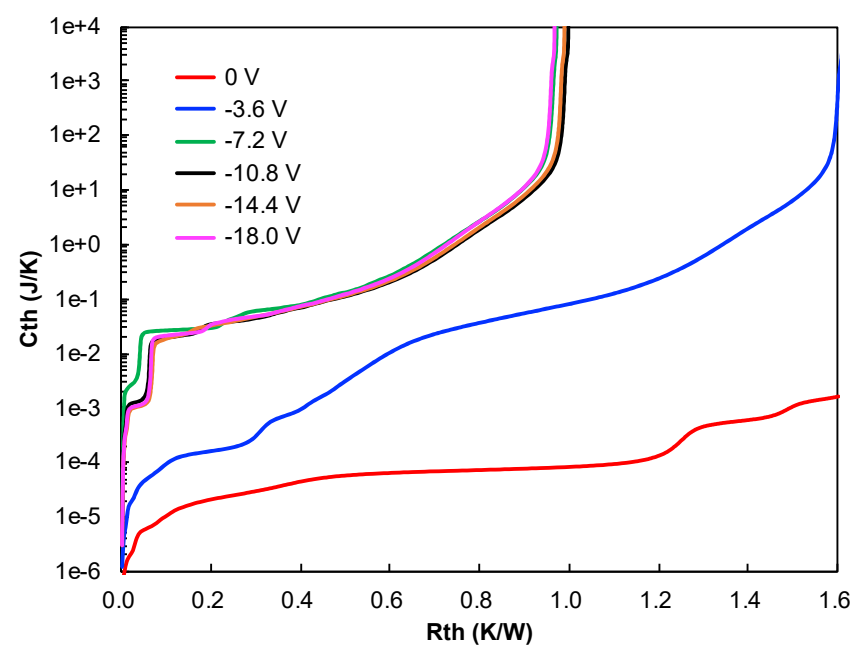

Fig. 11 Structure functions of Device B1 for various gate biases.

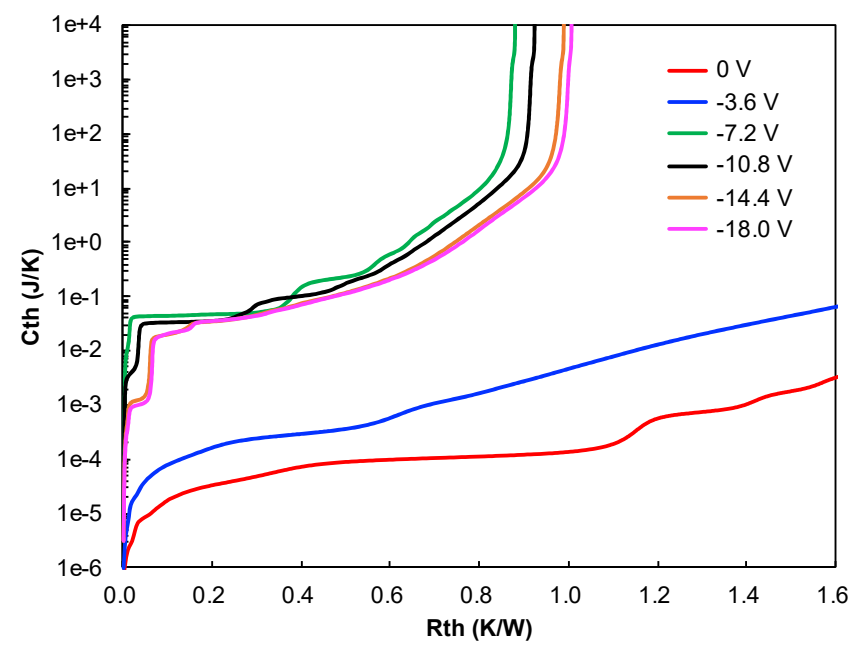

Fig. 12 Structure functions of Device B2 for various gate biases. 


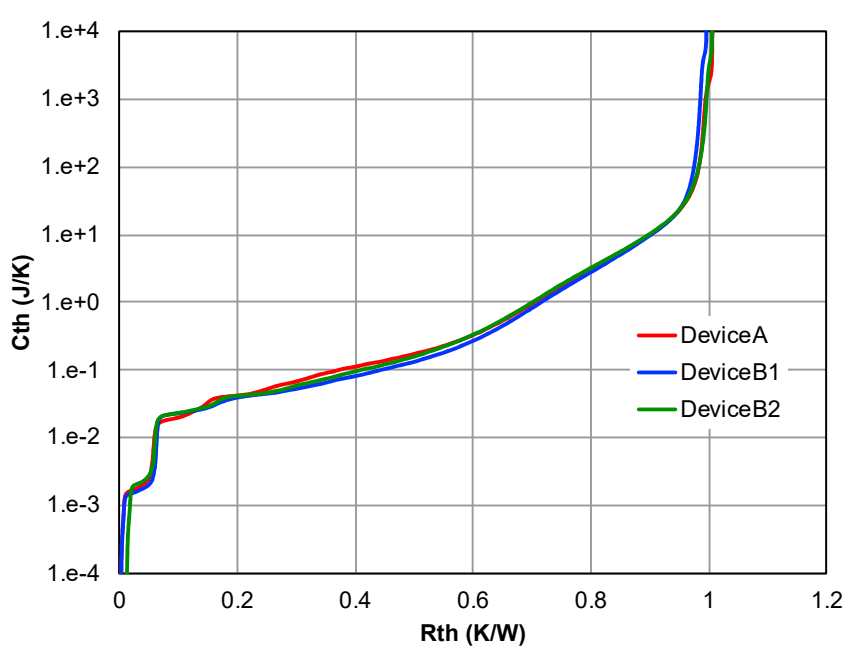

Fig. 13 Structure functions of Device A, Device B1, and Device B2.

expected. Similarly, the structure function graph also overlapped when the gate bias values were -14.4 and $-18.0 \mathrm{~V}$, where the transient temperature graph overlapped with device B2 (see Figs. 9 and 12).

Figure 13 shows the structure function graph of the applied gate bias values of $-9.8,-18.0$, and $-18.0 \mathrm{~V}$ for Device A, Device B1, and Device B2, respectively. The three structure function graphs of the devices are in good agreement. It is thought that the variation of the graph where the thermal resistance is up to $0.05 \mathrm{~K} / \mathrm{W}$ is attributed to measurement noise. The difference in the thermal resistance is $0.8 \mathrm{~K} / \mathrm{W}$ or more, and this results from a variation due to the installation state of the module to the cooling equipment. The difference between the graphs of Device B and Device A for $0.05 \mathrm{~K} / \mathrm{W}$ and $0.6 \mathrm{~K} / \mathrm{W}$ may arise from the differences in device thickness and active area size.

These results show that Vg mainly influences the transient temperature measurement of the device and that transient temperature measurement and TRAIT can be appropriately performed by setting the appropriate $\mathrm{Vg}$.

\section{Conclusion}

Accurate transient thermal analysis depends on the measurement accuracy of the transient temperature of the device. In order to accurately measure the transient temperature, it is important to set the measurement condition so that the temperature-voltage characteristics of the device are one to one. An appropriate gate bias is necessary for using the $\mathrm{SiC}-\mathrm{MOSFET}$ body diode as a temperature sensor. A method to set the appropriate $\mathrm{Vg}$ is proposed herein. This method employs the $\mathrm{Vg}-\mathrm{Vsd}$ characteristic and its first derivative. We confirmed that the transient temperature measurement and transient thermal analysis can be carried out by choosing Vg in the plateau region of the $\mathrm{Vg}-\mathrm{Vsd}$ characteristic. By applying this method, the accurate thermal temperature (or thermal impedance) of the $\mathrm{SiC}$ power module package can be measured. Therefore, a more accurate thermal structure function of the $\mathrm{SiC}$ power module package can be obtained. This method is useful for verifying package thermal design, reliability evaluation, and product quality evaluation. We believe it will help improve the power density of $\mathrm{SiC}$ power modules and achieve stable and reliable operation. In addition, this method is considered useful not only for determining the parameters of transient temperature measurement for SiC-MOSFET, but also for determining the measurement parameters for unknown transistors.

\section{Acknowledgment}

This work was supported by Council for Science, Technology and Innovation (CSTI), Cross-ministerial Strategic Innovation Promotion Program (SIP), "Next-generation power electronics/Consistent R\&D of next-generation SiC power electronics" (funding agency: NEDO).

\section{References}

[1] S. Abedinpour and K. Shenai, "Power electronics technologies for the new millennium,” IEEE Third International Caracas Conference on Devices, Circuits, and Systems, IEEE Catalog Number: 00TH8474C, pp. P111-1-P111-9, 2000.

[2] K. Sasaki, S. Sato, K. Matsui, Y. Murakami, S. Tanimoto, and H. Tanisawa, " $40 \mathrm{~kW} / \mathrm{L}$ high switching frequency three-phase AC $400 \mathrm{~V}$ all-SiC inverter," Materials Science Forum, Vol. 740-742, pp. 10811084, 2013

[3] F. Kato, R. Simanjorang, F. Lang, H. Nakagawa, H. Yamaguchi, and $\mathrm{H}$. Sato, " $250^{\circ} \mathrm{C}-$ Operated sandwich-structured all-SiC power module," Japanese Journal of Applied Physics, Vol. 54, 04DP06, 2015.

[4] S. Sato, H. Tanisawa, K. Kohi, H. Takahashi, Y. Murakami, F. Kato, K. Watanabe, and H. Sato, "Switching analysis for a full $\mathrm{SiC}$ module," The European Conference on Silicon Carbide and Related Materials, ECSCRM2016.

[5] V. Szekely, "A new evaluation method of thermal transient measurement results," Microelectronics Journal, Vol. 28, No. 3, pp. 277-292, 1997.

[6] JEDEC standard, JESD51-14, "Transient dual inter- 
face test method for the measurement of the thermal resistance junction to case of semiconductor devices with heat flow through a single path,” JEDEC Solid State Technology Association, 2010.

[7] JEDEC standard, JESD51-1, "Integrated Circuits thermal measurement method - electrical test method (Single semiconductor device)," JEDEC Solid State Technology Association, 1995.

[8] T. Funaki and S. Fukunaga, "Difficulties in characterizing transient thermal resistance of SiC MOSFETs," Therminic, pp. 141-146, 2016.

[9] F. Kato, et al., "Effect of forward voltage change depending on gate voltage in body diode of SiCMOSFET at thermal transient testing for analysing SiC power module package," Therminic, 2017, pp. $1-4$.
[10] A. J. Lelis, et al., "Time dependence of bias-stressinduced SiC MOSFET threshold-voltage instability measurements," IEEE Transactions on Electron Devices, Vol. 55, No. 8, pp. 1835-1840, 2008.

[11] P. E. Bagnoli, C. Casarosa, et al., "Thermal resistance analysis by induced transient method (TRAIT) applied to power electronic device packaging," In: EPEÕ 95 Conference Proceedings, Seville, Vol. 3, pp. 322-327, 1995.

\section{Fumiki Kato}

Shinji Sato

Hidekazu Tanisawa

Hiroshi Hozoji

Hiroshi Yamaguchi

Hiroshi Sato 